\title{
Efficacy of low-dose imatinib in chronic-phase chronic myelogenous leukemia patients
}

\author{
S. Kobayashi • F. Kimura • A. Kobayashi $•$ K. Sato • \\ K. Motoyoshi
}

Published online: 4 October 2008

(C) Springer-Verlag 2008

\section{Erratum to: Ann Hematol \\ DOI 10.1007/s00277-008-0589-2}

The presentation of Table 1 was incorrect. The correct version is given below.

Table 1 Baseline characteristics of patients

\begin{tabular}{llll}
\hline Characteristic & Patients $(\mathrm{n}=34)$ & $300 \mathrm{mg} / \mathrm{day}(\mathrm{n}=17)$ & $\geq 400 \mathrm{mg} / \mathrm{day}(\mathrm{n}=17)$ \\
\hline $\begin{array}{l}\text { Age at the time of imatinib initiation, years } \\
\text { Median }\end{array}$ & 55 & 56 & 55 \\
Range & $31-77$ & $34-77$ & $31-67$ \\
Male, number (\%) & $26(76.5)$ & $11(64.7)$ & $15(88.2)$ \\
Sokal score, number (\%) & & & \\
Low & $10(29.4)$ & $4(23.5)$ & $6(35.3)$ \\
Intermediate & $11(32.4)$ & $5(29.4)$ & $6(35.3)$ \\
High & $13(38.2)$ & $8(47.1)$ & $5(29.4)$ \\
Time from diagnosis to imatinib initiation, months & & 2 & 1 \\
Median & 1 & $0-81$ & $0-56$ \\
Range & $0-81$ & $5(14.7)$ & $3(8.8)$ \\
Treatment history, number (\%) & & $2(5.9)$ & $1(2.9)$ \\
Total & $8(23.5)$ & $3(8.8)$ & $2(5.9)$ \\
Hydroxyurea & $3(8.8)$ & $5(14.7)$ &
\end{tabular}

Funding and conflicts of interest No relevant conflicts of interest to declare.

Data from this study have not been previously published.

The online version of the original article can be found at http://dx.doi. org/10.1007/s00277-008-0589-2.

S. Kobayashi · F. Kimura • A. Kobayashi $\cdot$ K. Sato $\cdot$ K. Motoyoshi

Division of Hematology, Department of Internal Medicine,

National Defense Medical College,

3-2 Namiki,

Tokorozawa, Saitama 359-8513, Japan

K. Motoyoshi $(\square)$

Department of Internal Medicine,

National Defense Medical College,

3-2 Namiki,

Tokorozawa, Saitama 359-8513, Japan

e-mail: motoyosi@ndmc.ac.jp 